\title{
Norois
}

Environnement, aménagement, société

$219 \mid 2011$

Le restaurant, outil de développement local ?

\section{Revitalisation d'une économie locale et stratégie familiale : le cas Marcon à Saint-Bonnet-le-Froid}

Local economic revitalization and family strategy: the example of the Marcon family in Saint-Bonnet-le-Froid

\section{Vincent Marcilhac}

\section{(2) OpenEdition}

\section{Journals}

Édition électronique

URL : http://journals.openedition.org/norois/3592

DOI : 10.4000 /norois.3592

ISSN : $1760-8546$

\section{Éditeur}

Presses universitaires de Rennes

Édition imprimée

Date de publication : 30 juin 2011

Pagination : 41-56

ISBN : 978-2-7535-1479-9

ISSN : 0029-182X

\section{Référence électronique}

Vincent Marcilhac, «Revitalisation d'une économie locale et stratégie familiale : le cas Marcon à SaintBonnet-le-Froid », Norois [En ligne], 219 | 2011, mis en ligne le 30 septembre 2013, consulté le 01 mai 2019. URL : http://journals.openedition.org/norois/3592 ; DOI : 10.4000/norois.3592

Ce document a été généré automatiquement le 1 mai 2019.

(c) Tous droits réservés 


\title{
Revitalisation d'une économie locale et stratégie familiale : le cas Marcon à Saint-Bonnet-le-Froid
}

\author{
Local economic revitalization and family strategy: the example of the Marcon \\ family in Saint-Bonnet-le-Froid
}

Vincent Marcilhac

1 Comme son nom l'indique, Saint-Bonnet-le-Froid, situé à 1150 mètres d'altitude, est d'abord connu pour les conditions climatiques rigoureuses des hivers enneigés qui en font une étape traditionnelle du Rallye automobile Monte-Carlo. Mais ce village de 250 habitants est aussi célèbre grâce au chef Régis Marcon, dont le restaurant est classé trois étoiles au Guide Michelin depuis 2005. Pourtant, les restaurants triplement étoilés sont historiquement implantés dans les villes importantes (Paris et Lyon notamment) et le long des grands axes de circulation, formant une «diagonale gourmande " (Bailly et Hussy, 1991) entre Paris et la Côte d'Azur. Cette répartition géographique des «grandes tables » est complexe à expliquer: la densité de population, l'urbanisation, les axes de transport majeurs, la proximité d'autres restaurants réputés, la concentration de curiosités historiques ou géographiques sont autant de variables explicatives qui complètent les critères de créativité et de qualité des plats, du service et du cadre. L'essor de l'automobile et des loisirs au $\mathrm{xx}^{\mathrm{e}}$ siècle a également contribué à dessiner la carte de France des grands restaurants (fig. 1) : comme l'a écrit C. Fischler (2001), «Il existe un lien historique indissoluble entre l'automobile, le Guide Michelin et la grande cuisine. Les grands restaurants du guide rouge sont en général situés le long des grands axes routiers. Dès 1935, la répartition géographique des 3 étoiles est remarquablement concentrée. Trois grandes régions, historiquement, se taillent la part du lion: Paris, la Bourgogne, mais surtout ce qu'on appelle aujourd'hui la région Rhône-Alpes. La galaxie des trois étoiles s'étend donc du Nord vers le Sud, le long d'un axe Paris-Lyon-vallée du Rhône, le long surtout des nationales 6 et 7. La densité est beaucoup moins forte à l'Ouest d'une ligne Paris-Toulouse. Après la guerre, la dissémination des trois étoiles se poursuit vers le 
Sud : à partir de 1955, la Provence, puis la Côte d'Azur s'étoilent de plus en plus, suivant ainsi les itinéraires de plus en plus fréquentés su soleil et des vacances automobiles. »

Quelques grands chefs ont pourtant choisi d'implanter délibérément leurs établissements dans des zones rurales. Ils sont de plus en plus nombreux au cours de ces trente dernières années à l'avoir fait. L'époque où André Pic décidait de fermer son restaurant L'Auberge du Pin près de Saint-Péray en Ardèche pour s'installer à Valence semble bien révolue (Mesplède, 2004). En effet, depuis les années 1970, on observe que certains chefs, comme Michel Guérard ou plus tard Michel Trama, ont quitté Paris pour s'implanter dans des zones rurales; d'autres ne les ont jamais quittées : c'est le cas de Michel Bras ou de Régis Marcon. Alors que les cuisines régionales sont de moins en moins représentées sur les grandes tables de Paris et de province, ces chefs prennent le contre-pied de la tendance générale en revendiquant un localisme culinaire.

Figure 1 : Carte des restaurants ayant obtenu trois étoiles au Guide Michelin durant plus de dix ans/Map of restaurants which were awarded three stars by the Michelin Guide for more than ten years

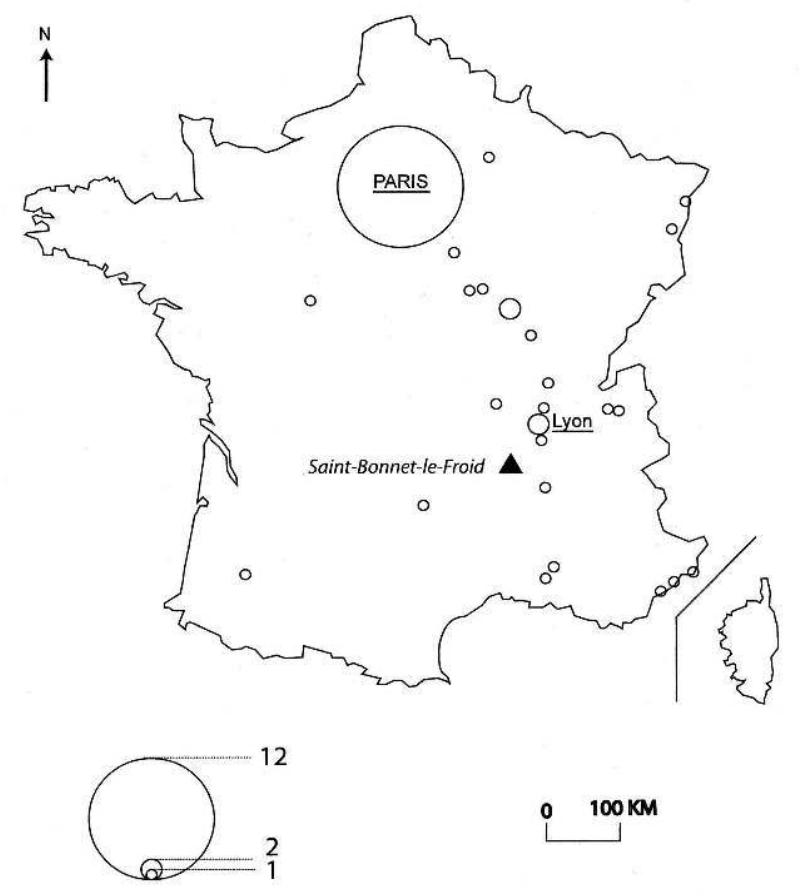

Dans quelle mesure le restaurant de Régis Marcon a-t-il impulsé une dynamique de développement local fondée sur la spécialisation du village dans le tourisme gourmand? Quel rôle joue la famille Marcon dans la stratégie visant à faire du village une destination touristique gourmande?

Pour répondre à ces questions, nous verrons d'abord que cette dynamique de développement local s'est mise en place dans un cadre géographique spécifique, un village situé sur un col où le passage a favorisé les activités liées au commerce et à l'accueil. Nous montrerons ensuite que cette dynamique est née de l'initiative privée celle du chef Régis Marcon - et s'inscrit dans une stratégie familiale, combinant les actions de chacun dans un but déterminé : spécialiser le tissu économique local dans les commerces de bouche et le tourisme gourmand. Enfin, nous démontrerons que cette 
dynamique nécessite la mobilisation des habitants du village derrière le chef et sa famille. Ainsi, les réseaux de solidarité entre les acteurs locaux sont entretenus par les liens commerciaux, la vie associative et les fêtes gourmandes.

5 Notre travail s'est appuyé sur les réponses de Régis Marcon à un questionnaire adressé en avril 2008, suivi d'un entretien avec le chef en juillet 2009. Notre questionnaire sur son restaurant était constitué de trois séries de questions. La première série portait sur le choix de la localisation et de l'agencement du restaurant ; la deuxième sur la composition $\mathrm{du}$ personnel et sur la clientèle; la troisième enfin sur les produits (provenance, fournisseurs), les plats proposés et la cave à vins (nombre de bouteilles, les régions viticoles françaises les plus représentées, les vins étrangers les plus représentés, la fourchette des prix). L'entretien a permis de préciser certains points du questionnaire (liste des fournisseurs) et surtout de mieux comprendre les rapports du chef avec les autres acteurs locaux. Cette enquête a été complétée par un entretien avec le négociant en vin Johannès Marcon, neveu du chef, à la Cave Marcon de Saint-Bonnet-le-Froid. Nous avons également enquêté auprès d'une vingtaine d'habitants de Saint-Bonnet-le-Froid, à l'office de Tourisme de Montfaucon et à son antenne de Saint-Bonnet-le-Froid, à l'Agence Locale de Tourisme du Pays de la jeune Loire, pour mieux comprendre le regard porté par les habitants et les acteurs du tourisme sur le restaurant de Régis Marcon et son rôle dans la dynamique de développement local fondé sur le tourisme gourmand.

\section{Un restaurant de renommée internationale installé dans un espace rural isolé}

6 Au premier abord, Saint-Bonnet-le-Froid peut apparaître comme une commune enclavée : le village de 250 habitants est localisé à plus de 1000 mètres d'altitude et à l'écart des grands axes de circulation, d'où son isolement relatif lors des hivers enneigés. Ces conditions expliquent la fermeture du restaurant étoilé durant la période hivernale et participent à la saisonnalité des activités touristiques. Mais, à y regarder de plus près, son site et sa situation sont moins défavorables qu'en apparence.

\section{Saint-Bonnet-le-Froid : site et situation}

7 Saint-Bonnet-le-Froid est en effet situé à proximité du Col des Baraques où la présence d'aubergistes et de commerçants est ancienne: «Saint-Bonnet a toujours été un lieu de passage » selon Johannès Marcon. Cette ouverture du village sur l'extérieur a favorisé l'esprit d'entreprise : c'est un élément de l'identité socioculturelle, qui a été un atout dans le processus de développement local (Matteudi, 1997). La fréquentation de ce lieu de passage a été favorisée par la proximité du centre de pèlerinage de Lalouvesc ${ }^{1}$, comme en témoignent les cartes postales du village au début $d u x^{e}$ siècle, où est indiquée la mention «environs de Lalouvesc ». De plus, il est au contact du plateau ardéchois et du massif du Mézenc, des provinces historiques du Velay et du Vivarais, à proximité de l'axe rhodanien et du pays stéphanois. Enfin, un réseau urbain équilibré maille l'espace dans un rayon d'une centaine de kilomètres autour du village, avec des villes de moins de 20000 habitants (Annonay, Yssingeaux), des villes de 20000 à 100000 habitants (Le Puyen-Velay et Valence), une ville de plus de 100000 habitants (Saint-Étienne) et une ville de plus d'un million d'habitants (Lyon), l'axe majeur rhodanien étant situé à une quarantaine de kilomètres (fig. 2). 


\section{Le restaurant étoilé : un fort marqueur territorial}

L'établissement de Régis Marcon appartient à la catégorie des restaurants luxueux ${ }^{2}$, dont la localisation n'est pas aléatoire, mais correspond à un marqueur territorial, c'est-à-dire un «objet situé dans un espace et un signe symbolique connotant ce lieu du fait de la clientèle » (Bailly et Paelinck, 1990). La réputation gastronomique construite entre 1995 (année où Régis Marcon remporte le trophée du concours international de cuisine du Bocuse d'Or) et 2005 (année de l'obtention de la troisième étoile au Guide Michelin) et le choix d'un nouveau site pour le restaurant étoilé, devant apparaître comme «exceptionnel» par sa proximité géographique avec la «nature ${ }^{3}$ ", donnent de la puissance au marqueur territorial, attirant une clientèle ciblée à haut pouvoir d'achat prête à parcourir des distances importantes pour s'y rendre (Knafou, 2008).

9 La clientèle de Régis Marcon est principalement française, en provenance surtout de la région Rhône-Alpes (30 \%), d'Auvergne (10\%) et de la région parisienne (10 \%). Les clients étrangers sont majoritairement originaires de quatre pays : l'Angleterre, la Suisse, la Belgique et le Japon. La clientèle est surtout composée de couples, âgés de 40 à 60 ans, issus de catégories socioprofessionnelles supérieures ("professions libérales", «artistes», etc.). Selon le questionnaire rempli par Régis Marcon au printemps 2008, cette clientèle serait la même au déjeuner et au dîner.

10 Si la localisation du restaurant étoilé dans le village de Saint-Bonnet-le-Froid peut apparaitre a priori comme un handicap, Régis Marcon est parvenu à en faire un lieu symbolique $^{4}$ grâce à sa réputation et à ses investissements (tant en infrastructures qu'en hommes).

\section{L'entreprise Marcon : du restaurant étoilé aux établissements multiples}

11 La dynamique de développement local est le résultat d'une stratégie familiale, qui a su mobiliser les acteurs locaux, publics et surtout privés, autour d'un même projet fondé sur une spécialisation du tissu économique local, renforçant l'attractivité touristique de Saint-Bonnet-le-Froid grâce à la réputation du restaurant triplement étoilé. 


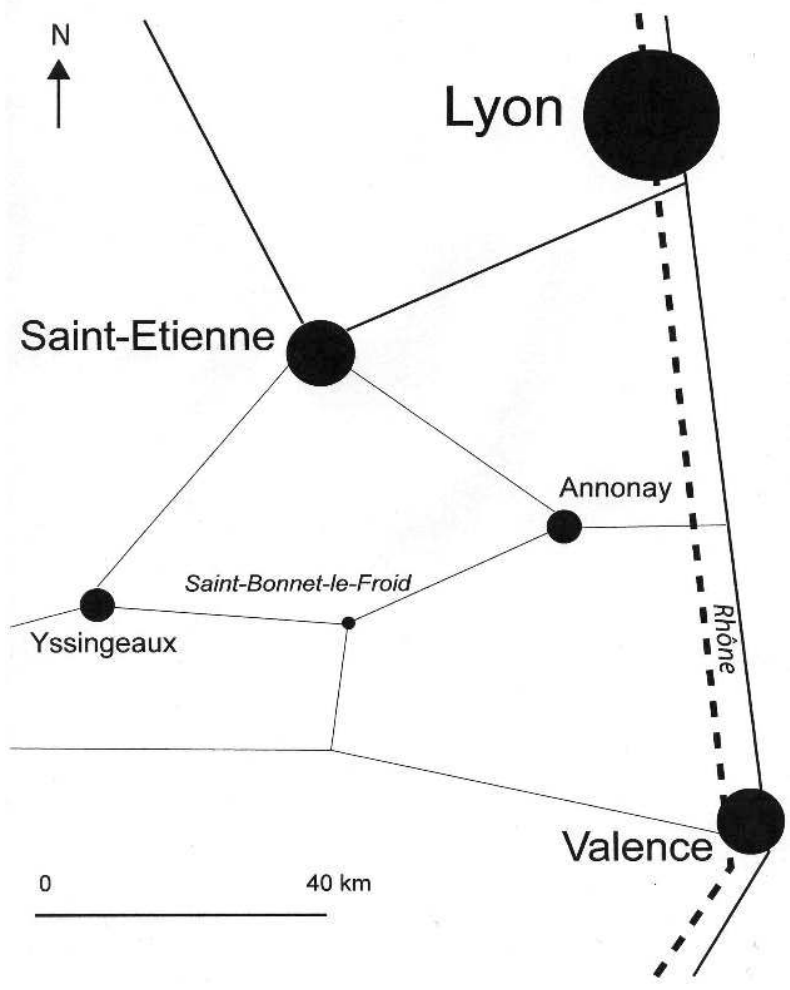

\section{L'ancrage local de la famille Marcon}

Expliquer par la géographie l'implantation du restaurant étoilé de Régis Marcon à SaintBonnet-le-Froid serait sans doute insuffisant ; c'est davantage dans son attachement à son village natal et à ses habitants qu'on en trouve la cause ${ }^{5}$. L'ancrage territorial, désignant le sentiment d'appartenance à un lieu, est donc un facteur majeur d'explication de l'initiative entrepreneuriale de Régis Marcon dans son village natal : « Le village, c'est une petite famille ${ }^{6}$.» En 1948, la famille Marcon s'installe à Saint-Bonnet-le-Froid, développant à la fois l'activité d'aubergiste et celle de marchand de vins. Après la mort de ses parents, Régis a repris avec son épouse (fille d'hôteliers de Lalouvesc) l'hôtelrestaurant familial (photo 1), l'Auberge des Cîmes (aujourd'hui rebaptisée «Bistrot la Coulemelle»), qui a abrité jusqu'en 2005 le restaurant étoilé, tandis que son frère Guy a poursuivi l'activité de commerce du vin. Ses deux autres frères, André et Jean-Pierre, sont aussi très impliqués dans la vie politique et économique tant à l'échelle locale qu'à l'échelle nationale : André Marcon est maire du village (où il tient un hôtel) depuis 1989 et président de l'Assemblée des Chambres Françaises de Commerce et d'Industrie après avoir dirigé la Chambre de Commerce et d'Industrie du Puy-en-Velay puis la Chambre Régionale de Commerce et d'Industrie d'Auvergne ; Jean-Pierre Marcon est député de la circonscription. La famille Marcon est donc bien enracinée localement. 
Photo 1 : L'Auberge des Cimes, du restaurant de luxe au restaurant familial (cl. V. Marcilhac, juillet 2009)/L'Auberge des Cimes, from luxury restaurant to family restaurant

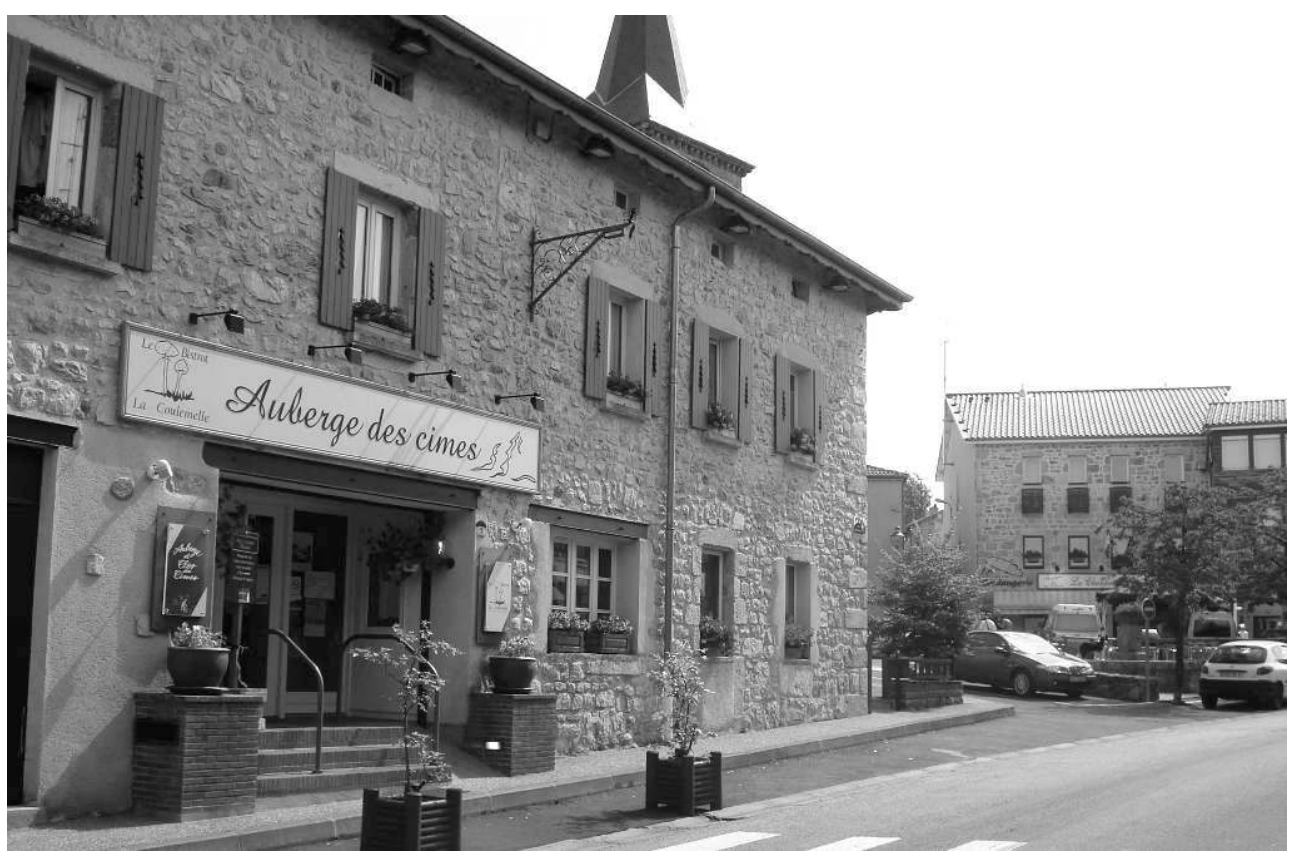

Le projet de développement local par le tourisme gourmand correspond d'abord à une stratégie familiale : les frères Marcon (Régis, Guy, André, Jean-Pierre), par leurs activités professionnelles ou leurs mandats, ont favorisé la revitalisation de l'économie locale de leur village par un projet original de développement par le tourisme gourmand. À l'échelle de l'entreprise Marcon, la stratégie repose essentiellement sur le couple: Régis en cuisine, Michèle en salle. Depuis que son fils aîné, Jacques, a rejoint sa brigade de cuisine en 2005, année de la consécration avec l'obtention de la troisième étoile au Guide Michelin, Régis Marcon est entré dans une stratégie patrimoniale, sans laquelle les investissements colossaux (près de 3 millions d'euros) réalisés dans la construction du nouvel hôtel-restaurant «Régis \& Jacques Marcon» n'auraient pas de sens : il s'agit en effet de pérenniser l'affaire familiale, en assurant la continuité de père en fils. En 2008, son second de cuisine, Éric Pras, est parti en Bourgogne au restaurant Lameloise, triplement étoilé au Guide Michelin, pour y succéder en cuisine à Jacques Lameloise un an plus tard. Jacques Marcon a alors pris la place d'Éric Pras dans la brigade de son père à Saint-Bonnet-le-Froid.

\section{L'agrandissement de l'entreprise : une évolution nécessaire pour rentabiliser le restaurant étoilé}

Aujourd'hui, le poids économique et social de la famille Marcon à Saint-Bonnet-le-Froid est considérable: Régis Marcon est propriétaire de deux hôtels de standing, trois et quatre étoiles (le deuxième ouvert en 2008), deux restaurants (le deuxième ouvert en 2005), une pâtisserie-salon de thé en 2000 à laquelle s'est ajoutée une boulangerie en 2004, et un village de vacances. Au total, cela représente plusieurs dizaines d'emplois, dont une part importante dans le restaurant étoilé (la brigade de cuisine est composée d'environ vingt-cinq personnes et la brigade de salle d'une petite vingtaine d'employés). Ce modèle, basé sur la multiplicité des établissements dans un même lieu, est inspiré historiquement 
par les exemples de Michel Guérard à Eugénie-les-Bains (Landes) et de Georges Blanc à Vonnas (Ain). Michel Bras dans l'Aubrac a été une source d'inspiration pour le site et l'architecture de son établissement étoilé (Etcheverria, 2004) ainsi que pour certains produits dérivés comme le couteau présentant sa signature, tout comme Olivier Roellinger à Cancale pour la boulangerie-pâtisserie-salon de thé (Grain de Vanille), son potager, son école de cuisine "Corsaire ", ses "gittes marins" et son «cottage Les Rimains ». Cet agrandissement permet à Régis Marcon de réduire les risques liés à la mono-activité et de prolonger la durée du séjour de la clientèle grâce à des infrastructures d'hébergement de haut standing (hôtels 3 et 4 étoiles), tandis que les équipements tels que son parcours botanique et la boutique installée dans la boulangeriepâtisserie-salon de thé répondent aux attentes du touriste ${ }^{7}$ (Knafou, 2002). Néanmoins, face à l'endettement consenti pour de telles infrastructures, le modèle de développement mis en place est fragile : la perte d'une étoile au Guide Michelin ou l'aggravation de la crise économique actuelle auraient un impact certain sur la fréquentation de l'hôtel et du restaurant Régis \& Jacques Marcon, même si la structure de sa clientèle (principalement française et de provenance régionale) et l'absence de restaurants de même standing dans un rayon de 70 kilomètres par la route permettraient de limiter les dommages.

À la différence d'autres grands chefs français qui ont ouvert des établissements à l'étranger (États-Unis et Japon principalement), Régis Marcon a concentré ses investissements dans son village, en optimisant la rentabilité de son personnel. Ainsi Christophe Gasper est à la fois responsable du pain et des sucrés des deux restaurants et de la boulangerie-pâtisserie où sont vendus les produits signés «Régis Marcon » («la confiture de lentilles vertes du Puy " ou "le chocolat praliné aux cèpes ", mais aussi les livres de recettes, la vaisselle ou le couteau Régis Marcon).

\section{Mobilité et implantation du personnel du restaurant étoilé}

16 Force est de constater que la mobilité géographique est un élément important dans l'apprentissage des jeunes chefs pour qui l'itinéraire professionnel est marqué par le passage dans les brigades des restaurants étoilés. Si nous observons cette mobilité dans la brigade de Régis Marcon, nous constatons néanmoins une certaine stabilité parmi ses principaux collaborateurs malgré les départs des seconds de cuisine, Serge Vieira en 2006, Éric Pras en 2008 puis Edouard Mignot en 2010. Ils ont d'ailleurs fait en général un parcours classique : outre son fils (formé notamment chez Frédy Girardet en Suisse), les principaux piliers de sa brigade de cuisine, à l'exemple de Gwenaël Forot (originaire d'Ardèche), sont passés dans des écoles hôtelières et chez des grands chefs avant d'arriver à Saint-Bonnet-le-Froid. Si parfois certains sont originaires des régions limitrophes, comme le maitre d'hôtel stéphanois Cédric Servain ou son ancien second de cuisine clermontois Serge Vieira (aujourd'hui chef d'un établissement étoilé à Chaudes-Aigues, dans le Cantal), ils viennent en général de régions plus lointaines, ce qui n'empêche pas leur implantation: ainsi, l'ancien sommelier de Régis Marcon, travaille désormais à la Cave Marcon aux côtés du frère et des neveux du chef, et il a épousé sa nièce en 2008. Sur les cinq naissances dans le village en 2008, trois impliquent des membres de la famille Marcon, et dans deux cas, les pères sont des collaborateurs de Régis Marcon. D'autres employés sont devenus adjoints au maire. Cette implication dans la vie du village favorise l'implantation du personnel du restaurant étoilé à Saint-Bonnet-le-Froid, à l'exemple de Christophe Gasper et de Laurent Blanchon aux côtés de Régis Marcon depuis une 
décennie, et favorise une montée en gamme des commerces de bouche, soit au sein de l'entreprise de Régis Marcon à l'exemple de la boulangerie (photo 2), soit au sein d'un autre commerce comme la Cave Marcon.

Photo 2 : La boulangerie La Chanterelle au centre du village, ouverte en 2004 (cl. V. Marcilhac, juillet 2009)/“La Chanterelle”, Marcon bakery in the center of the village since 2004

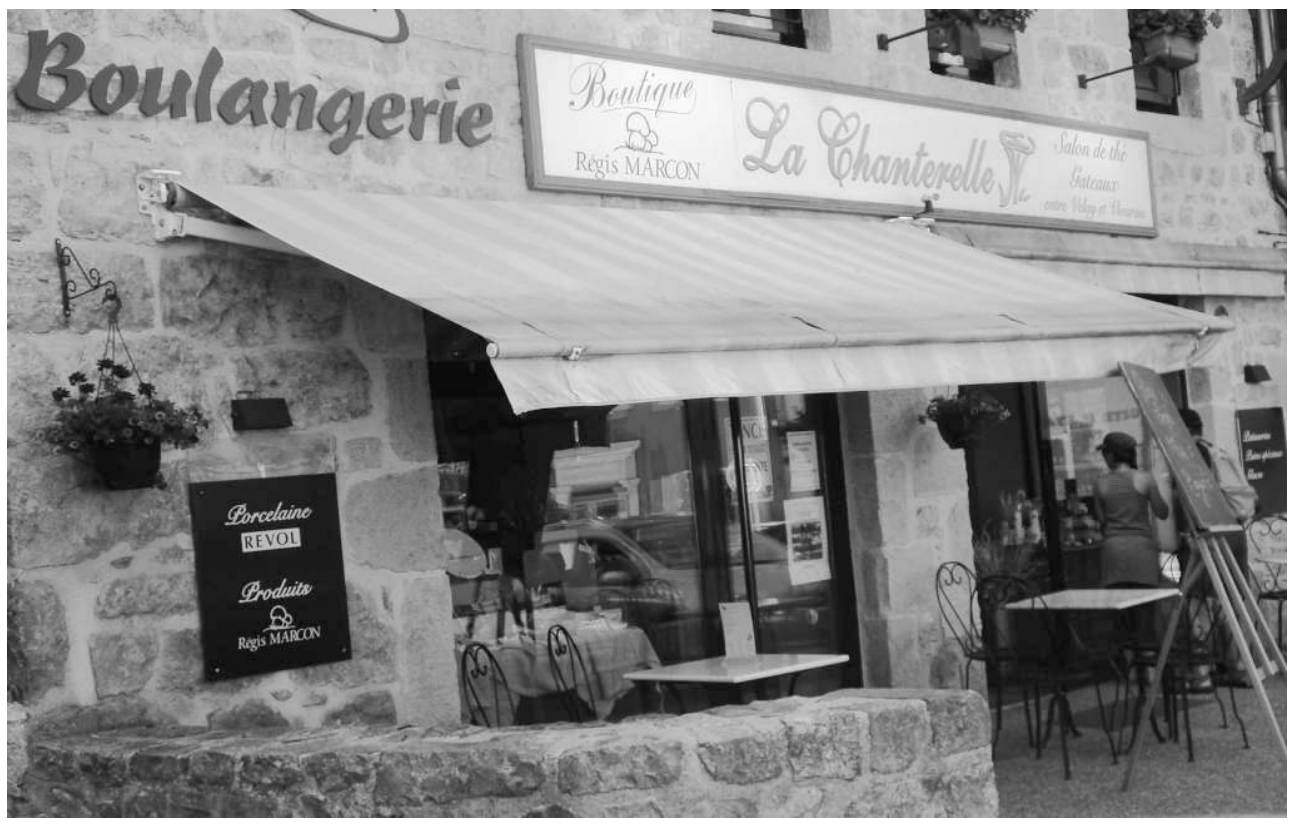

\section{Les réseaux de solidarité familiale}

Son frère Guy est propriétaire de la Cave Marcon, dont l'activité était jusque récemment destinée uniquement aux professionnels (cavistes, restaurateurs). Depuis 2006, Guy a toutefois développé des boutiques ouvertes aux clients et aux touristes, et qui proposent une offre diversifiée (plus de 1500 références, dont des produits étrangers comme du whisky japonais), d'abord au Puy-en-Velay, puis à Saint-Bonnet-le-Froid et à SaintGenest-Malifaux, et dernièrement à Clermont-Ferrand. Il est l'un des fournisseurs privilégiés de son frère Régis pour la carte des vins ( 45000 bouteilles en cave, avec une préférence pour les vins de la vallée du Rhône), mais aussi des autres restaurants de la commune pour la clientèle desquels il élabore des coffrets. En même temps, il distribue les produits signés Régis Marcon, dans un espace réservé à l'épicerie fine. La troisième génération des Marcon, avec Jacques au restaurant étoilé depuis six ans ou Johannès, Maguy et Jean-Régis à la Cave Marcon, s'apprête à prendre la relève.

\section{Le produit local comme source d'innovation culinaire et de promotion territoriale}

18 Le produit local cuisiné et mis en scène dans le restaurant étoilé est un «emblème territorial » qui, «par métonymie, représente et même signifie cet espace et les valeurs qui lui sont attribuées » (Lévy et Lussault, 2003). En devenant une icône du restaurant étoilé et du village, le produit local constitue un outil de promotion territoriale. 
Pour analyser le discours du chef Régis Marcon sur sa cuisine, nous nous sommes appuyés sur l'analyse sémiologique de ses menus (printemps 2009), d'un lexique destinés aux dossiers de presse et aux clients de l'hôtel, et sur la formulation des réponses de Régis Marcon à notre questionnaire et lors de notre entretien. Nous nous sommes également appuyés sur des notes personnelles de Régis Marcon portant sur sa cuisine, sa famille, son village et ses projets, qu'il nous a remises lors de l'entretien. Ces notes ont servi à la rédaction de l'ouvrage intitulé La cuisine de Régis et Jacques Marcon (Mascon et al., 2009).

\section{La rhétorique culinaire de régis marcon}

20 Le « localisme » est au cœur du discours gastronomique de Régis Marcon, qui intègre dans sa démarche à la fois les enjeux sociaux (maintien et création d'activités en milieu rural), culturels (transmission des recettes culinaires locales et de plats régionaux) et environnementaux (usage de produits locaux et issus de l'agriculture biologique). L'hôtelrestaurant Régis \& Jacques Marcon, qui a obtenu le Certificat Ecolabel Européen, a été construit entre 2005 et 2008 en incluant trois volets dans sa démarche environnementale : l'intégration de l'établissement dans le paysage, que ce soit par l'usage des matériaux locaux (pierre volcanique, granite et bois) ou l'architecture inspirée de celle d'une ferme (maison de maitre, écurie, grange) avec les chambres de l'hôtel construites dans la roche ; l'économie d'énergie (géothermie, chaufferie à bois, panneaux solaires, ampoules à faible consommation, panneaux photovoltaïques et récupération de chaleur par l'utilisation d'un plafond filtrant en cuisine, etc.) et d'eau (économiseurs d'eau); le tri des déchets tant en cuisine que dans l'hôtel. Les produits locaux sont à voir dans les paysages qu'offre la vue panoramique du restaurant, à lire dans les menus, et enfin à goûter. Le discours a une importance considérable, car il s'agit, comme au théâtre, de raconter une histoire celle d'un terroir tant réel qu'imaginaire. Ainsi, Régis Marcon accueille ses hôtes en mettant à leur disposition un lexique intitulé «L'Esprit en quelques mots » avec vingttrois termes. Le lexique commence par «Ardèche et Auvergne : indéniablement ancré dans sa région, Régis Marcon s'en inspire sans cesse; il y puise l'essence même de sa cuisine en travaillant les produits locaux ${ }^{9}$ mais aussi en respirant un air qui le ressource ». Ce localisme culinaire, auquel il est explicitement fait référence dans les deux menus de son établissement qui portent des titres évocateurs, «Le Vellave » et «Entre Velay et Vivarais ", est raconté, à la manière d'un conte, où le chef est le narrateur : le discours que Régis Marcon utilise permet à la fois de comprendre comment il perçoit la contrée où il habite et comment il construit un système cohérent de signes, une représentation du terroir, au travers des plats proposés dans son menu, à l'exemple de la Galette de champignons de retour de cueillette et des Fromages d'Ardèche et d'Auvergne. L'un des plats emblématiques sur le plan rhétorique est L'agneau noir du Velay en croûte de foin de cistre $^{10}$, dont Régis Marcon écrit dans son lexique que « ça vient en direct de la nature »: la référence à une race ovine locale et à l'usage d'une herbe sauvage, la cistre, caractéristique du massif du Mézenc (et qui fait d'ailleurs la typicité de l'AOC de race bovine « fin gras du Mézenc ») reflète le discours gastronomique local de Régis Marcon.

\section{La valorisation culinaire des ressources alimentaires locales}

L'implantation du nouveau restaurant (photo 3), doté d'une salle de $350 \mathrm{~m}^{2}$ (60 places) et d'une cuisine spacieuse de $200 \mathrm{~m}^{2}$ au sommet de la colline qui surplombe le village permet 
aux clients d'avoir une vue panoramique à $160^{\circ}$ sur le Pic Lizieux et les sucs de l'Yssingelais avec, au loin, le Mont Mézenc. Le paysage est ainsi intégré dans la promotion du localisme alimentaire.

Photo 3 : Le nouvel établissement de Régis et Jacques Marcon (cl. V. Marcilhac, février 2009)/Régis and Jacques Marcon's new luxury hotel and restaurant

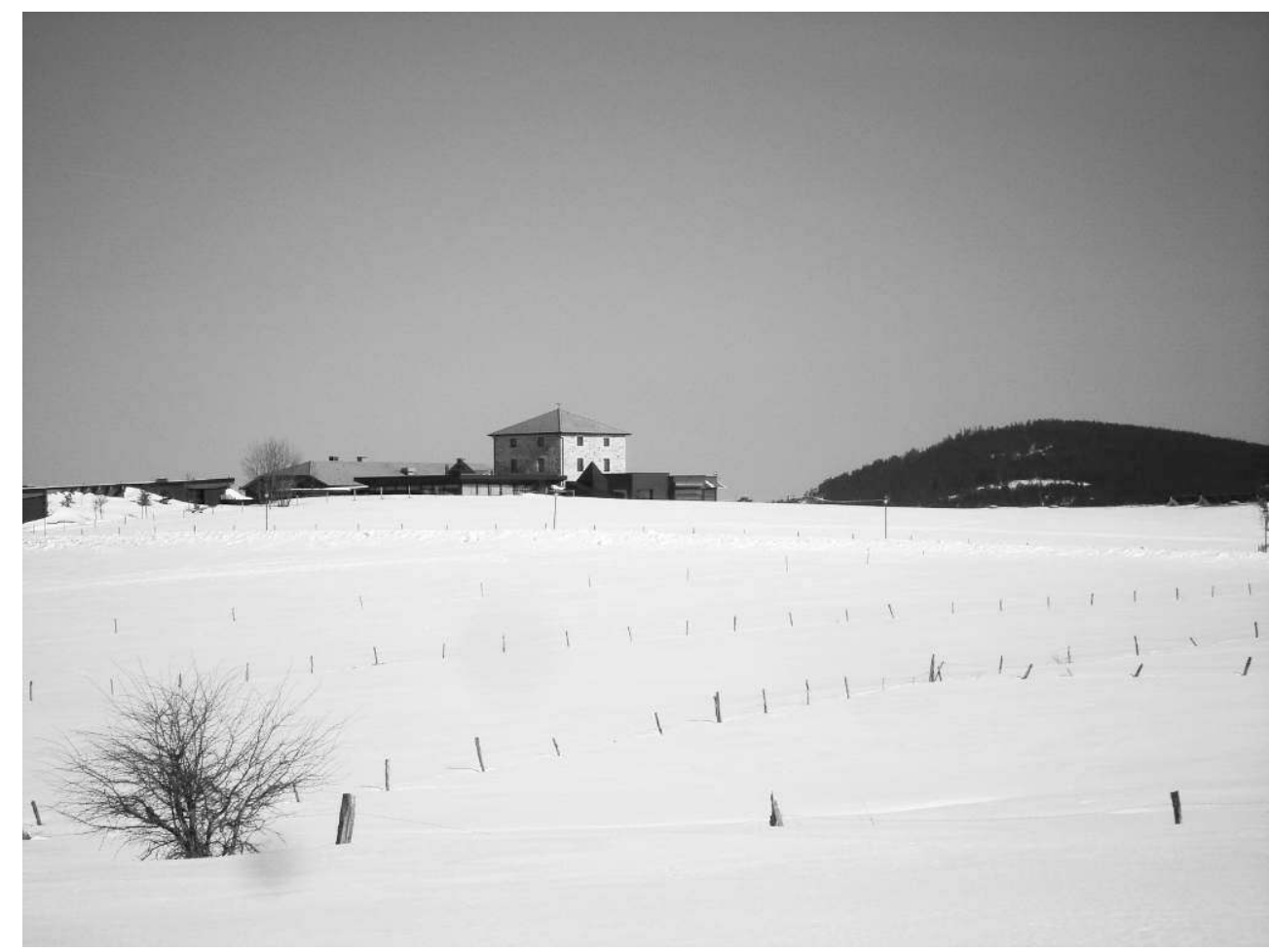

La valorisation des ressources alimentaires locales (Abert et André, 2000) se fait surtout par l'utilisation en cuisine d'ingrédients qui ont une provenance locale ou régionale (fig. 3) : la cueillette des fleurs et des plantes sauvages (cistre, verveine, etc.), des herbes (plantain, achillée, etc.) et des champignons ; la culture des légumes dans le jardin " bio » du restaurant ; les truites du Lignon (provenant de la pisciculture de Fay-sur-Lignon) ; les escargots d'Alain Charras à Grazac ; le miel de Vincent Faure à Fay-sur-Lignon (800 ruches à la Ferme des Abeilles); les fromages de chèvre de Daniel Mounier à Dunières; les fromages de la laiterie Gerentes à Araules (près d'Yssingeaux) ou de la ferme des Hautes Chaumes à Valcivières (fourme fermière) près d'Ambert; le poulet bourbonnais ; le bœuf du Mézenc ; la farine bio de la plaine du Forez; les huiles de Jean-Marc Montegottero à Beaujeu dans le Rhône ; les vins de la vallée du Rhône (en particulier ceux de Gérard et Jean-Louis Chave dans l'appellation Hermitage). La redécouverte de recettes locales ou régionales, comme la " brochette Margaridou ${ }^{11}$ » ou la potée auvergnate (servie avec du homard) s'inspire du discours régionaliste de l'entre-deux-guerres (Csergo, 1996) mettant en avant l'authenticité de la cuisine populaire et paysanne. 
Figure 3 : "Entre Velay et Vivarais ", l'invention d'une gastronomie locale/"Between Velay and Vivarais", the invention of a local gastronomy

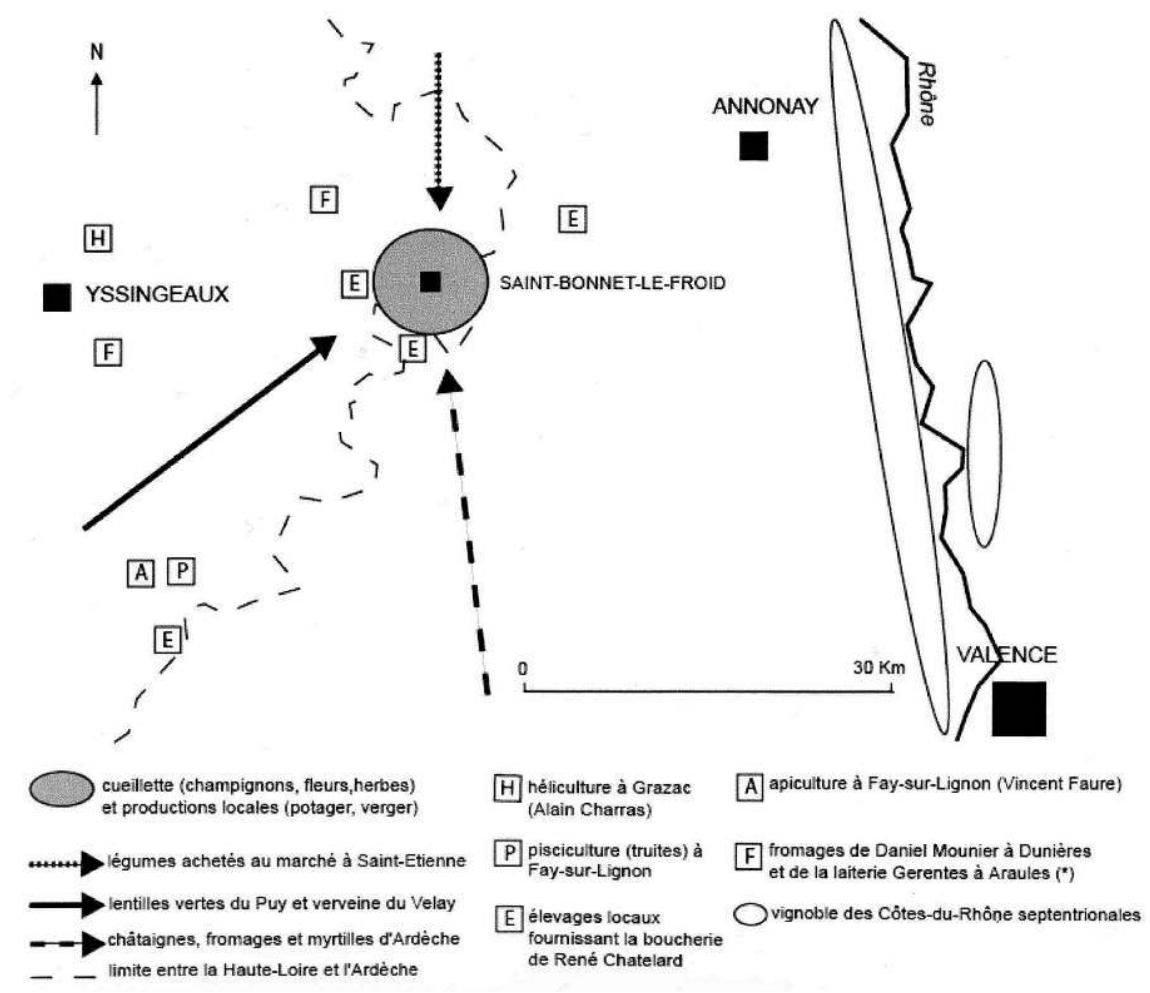

Grâce à la réputation gastronomique du chef étoilé, Saint-Bonnet-le-Froid est devenu un lieu connu et fréquenté par une clientèle de gourmets aisés, tant français qu'étrangers. Les acteurs locaux ont su utiliser l'image de marque du restaurant étoilé pour renforcer l'attractivité touristique du village et en faire une destination touristique gourmande.

\section{La spécialisation du village dans le tourisme gourmand}

La notion de «tourisme gourmand » (Lemasson, 2006) rend compte de l'évolution actuelle de l'ensemble des activités de temps libre et de loisirs dédiées à la découverte des produits alimentaires et à la manière de les apprêter, en mettant moins l'accent sur son caractère élitiste, et davantage sur la recherche de l'expérience sensorielle hédoniste. Surtout, l'évolution sémantique, du «tourisme gastronomique» au «tourisme gourmand", traduit une nouvelle place de l'alimentation dans le développement touristique, qui ne se limite plus à une fonction secondaire. La recherche de l'émotion gustative constitue, selon certains locuteurs médiatiques, l'objectif principal de nouvelles pratiques touristiques faisant du tourisme gourmand un moteur du développement économique à l'échelle locale. 


\section{Saint-Bonnet-le-Froid : lieu gourmand ou destination touristique gourmande?} secteurs de l'hôtellerie-restauration et du commerce alimentaire représentent environ 80 emplois et les taxes payées par les entreprises de ces secteurs correspondent à près de $15 \%$ du budget de fonctionnement de la commune en 2010. Depuis le début des années 2000, une antenne de l'Office de Tourisme de Montfaucon a ouvert à la mairie de SaintBonnet-le-Froid, proposant de découvrir « Saint-Bonnet-le-Froid, le village gourmand » à travers les brochures touristiques et les visites guidées. Un site internet attractif et fonctionnel ${ }^{12}$ met en avant le tourisme gourmand sur la communauté de communes du Pays de Montfaucon : la page d'accueil est intitulée « Haut Pays du Velay terre d'émotions et de gourmandises ", mettant ainsi en place une politique de marketing spatial (Beaudet, 2006) visant à promouvoir ce territoire comme une destination touristique gourmande. Surtout, l'Agence Locale de Tourisme (ALT) Pays de la jeune Loire, créée en 2006, est chargée de la stratégie de promotion touristique dans le réseau des Offices de Tourisme de l'est du département de la Haute-Loire. Le tourisme gourmand est, avec le tourisme vert, au cœur de sa stratégie de développement, comme en témoigne le slogan «Destination jeune Loire! Pour des vacances gourmandes et chlorophylle! » Financée par le Conseil régional d'Auvergne, l'Agence Locale de Tourisme assure la promotion de Saint-Bonnet-le-Froid comme une destination touristique gourmande via le réseau des offices de Tourisme qu'elle regroupe. Les campagnes de communication de L'ALT Pays de la jeune Loire ont ciblé principalement une clientèle du bassin lyonnais ${ }^{13}$ pour les deux temps forts lancés en 2010 : «les clés de la gourmandise » au printemps et «l'automne gourmand " entre octobre et décembre proposant des séjours sur un week-end durant l'un des six événements gourmands ${ }^{14}$ organisés dans la communauté de communes du Pays de Montfaucon, dont la foire aux champignons de Saint-Bonnet-le-Froid (6 et 7 novembre 2010) est l'événement majeur. L'objectif, ouvertement affiché, est de «valoriser des séjours à la charmante saison des champignons et pas seulement de l'excursionnisme à la journée ${ }^{15}$ ».

Le tourisme gourmand à Saint-Bonnet-le-Froid prend différentes formes: l'expérience gastronomique dans le restaurant triplement étoilé, ou dans l'un des trois autres restaurants de qualité distingués dans le Guide Michelin par un «bib gourmand » (le Fort du Pré, la Coulemelle, André Chatelard), la pratique de stages de cuisine durant l'année et à l'approche de Noël auprès des collaborateurs de Régis Marcon, la participation aux fêtes gourmandes de l'automne et du printemps, ou encore les ateliers « vins et fromages » à la Cave Marcon et la découverte de productions locales (miel, myrtilles, framboises, etc.) à l'exploitation durant la période estivale (sur réservation à l'office de Tourisme). L'office de Tourisme de Montfaucon, dont le président Jean-Pierre Santy a choisi depuis 2009 la découverte des produits de terroir et des métiers de bouche comme élément porteur du développement touristique ${ }^{16}$, et son antenne à la mairie du village organisent également des visites guidées payantes sur le thème "découvrez Saint-Bonnet-le-Froid, le village gourmand ", dont l'objectif est "d'expliquer, à travers l'histoire, pourquoi Saint-Bonnet-le-Froid est devenu un village gourmand ${ }^{17}$ »: «Il vous sera conté l'histoire de ses maisons qui sont devenues des restaurants renommés et des anecdotes sur ses villageois dont nos grands chefs sont les héritiers » indique le site internet de l'office de Tourisme. 
Ainsi, malgré la forte concentration de commerces de bouche, ceux-ci sont davantage partenaires que concurrents : par exemple, les frères Chatelard ${ }^{18}$, respectivement boucher (René) et restaurateur (André), sont pour l'un fournisseur des restaurants de Régis Marcon (et des autres restaurants du village), pour l'autre client de la Cave Marcon. Cette concentration exceptionnelle de commerces alimentaires haut-de-gamme (fig. 4) dans un petit village renforce indéniablement l'image gastronomique locale et favorise sa diffusion en suscitant la curiosité des médias locaux, régionaux et nationaux. Le maintien et la création d'activités autour des commerces alimentaires (une dizaine sur la quinzaine de commerces dans le village) ont enrayé le déclin démographique de Saint-Bonnet-leFroid, dont la population a augmenté de manière significative depuis vingt ans (200 habitants au début des années 1960, 180 habitants à la fin des années 1980, près de 250 habitants en 2008). Les naissances dépassent désormais les décès et le solde migratoire est positif, avec une population active qui représente $50 \%$ des habitants en 2008 et un taux de chômage de seulement 6 \%.

Figure 4 : Implantation des structures d'hébergement et commerces de bouche à Saint-Bonnet-leFroid/Map of accommodations and gourmet shops in Saint-Bonnet-le-Froid

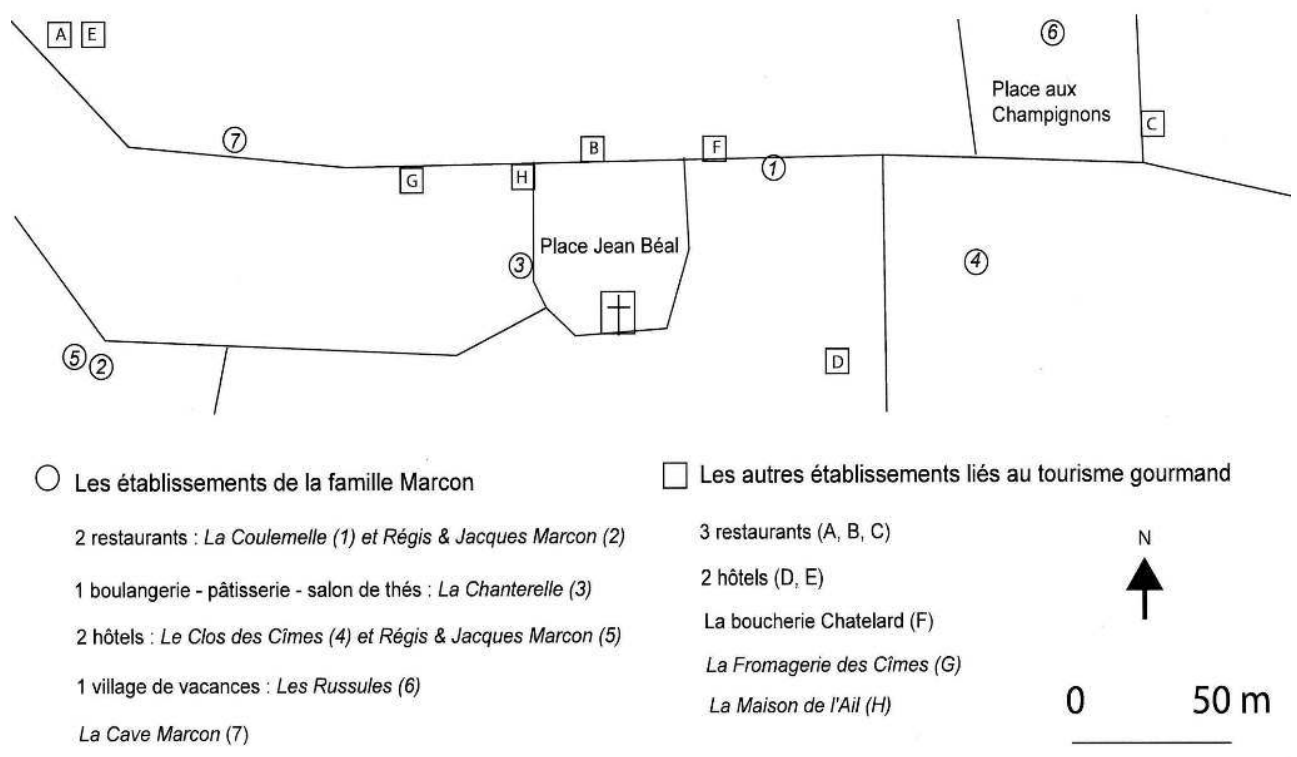

\section{Du réseau associatif au réseau touristique : une organisation réticulaire}

Si la vie associative tient une place importante dans la dynamique de développement local (Houée, 2001), Saint-Bonnet-le-Froid en est un exemple abouti. Le village compte une dizaine d'associations, parmi lesquelles un comité d'animation et une association des commerçants dirigés par Thierry Guyot, le chef-propriétaire du restaurant Le Fort du Pré, que Régis Marcon présente comme un partenaire. Le neveu de Régis Marcon, Jean-Régis, dirige l'association des jeunes, " le Club des Neiges ", qui organise notamment des sorties de ski de fond. L'implication de la famille Marcon dans la vie associative joue un rôle important dans la dynamique de développement local, car elle entretient entre la famille Marcon et les habitants du village des réseaux de solidarité (Pecqueur, 2000), qui sont de 
puissants vecteurs de mobilisation collective dans la stratégie de développement touristique (Violier, 2008).

Mais c'est surtout à l'échelle de la communauté de communes de Montfaucon, dans le cadre de l'Agence Locale de Tourisme du Pays de la jeune Loire (regroupant 6 communautés de communes dans les limites administratives du canton d'Yssingeaux), que la spécialisation dans le tourisme gourmand a été impulsée, en s'appuyant sur le " pôle gourmand ${ }^{19}$ » de Saint Bonnet-le-Froid. L'Office de Tourisme de Montfaucon a en effet un rôle de pilotage dans la stratégie de développement touristique basé sur la thématique du "Bon et Bien Manger», dont l'objectif est de promouvoir «une destination propice à la découverte des sens - de la terre à l'assiette - en toutes saisons ${ }^{20}$ ». Cette stratégie de développement touristique s'appuie sur une charte regroupant trois types d'acteurs locaux, appelés les «Ambassadeurs du Bon et du Bien Manger»: des producteurs, des artisans des métiers de bouche, des restaurateurs. Ces « ambassadeurs » accueillent des visiteurs lors des temps forts («les clés de la gourmandise » en avril, les journées du patrimoine en septembre, "l'automne gourmand» entre octobre et décembre), avec des goûters à la ferme, des ateliers de pâtisserie ou de boulangerie, des dégustations de produits locaux etc. Cette organisation réticulaire intègre également les acteurs politiques : «L'une des missions de l'Agence Locale de Tourisme est de développer une "culture tourisme" et de faire prendre conscience aux élus que le tourisme est un levier économique majeur ${ }^{21}$.»

\section{La construction récente d'une identité alimentaire locale}

Le champignon est à la fois l'emblème du village (« la place aux Champignons » est la place centrale du village) et celui de Régis Marcon, dont trois établissements portent des noms de variétés de champignons: Les Russules, La Chanterelle et La Coulemelle. C'est d'ailleurs avec le soutien actif de Régis Marcon que le comité d'animation de SaintBonnet-le-Froid a relancé au milieu des années 1990 la «Foire aux Champignons » attirant chaque année en début novembre plusieurs milliers de visiteurs (environ 15000 selon l'Office de Tourisme et le comité d'animation) dans le village. Durant deux jours, le village vit au rythme des champignons: "l'espace des producteurs" (marché aux champignons) et "l'espace gourmand (cours de cuisine, démonstration et repas aux champignons) sont regroupés sur la place aux Champignons tandis que les mycologues se rassemblent sur la place Jean Béal autour de l'exposition des champignons. Cet événement gourmand, qui mobilise plusieurs dizaines de bénévoles, attire des touristes venant de Haute-Loire, mais aussi des départements limitrophes. Les hôtels du village sont complets plusieurs mois à l'avance, à l'exemple du Fort du Pré qui, pour la Foire aux Champignons du 5 et 6 novembre 2011, est plein pour ces dates dès le début de l'été. La rue principale du village est alors rendue aux piétons et aux forains, tandis que différents événements festifs autour de produits et de plats régionaux (soupe aux champignons, tarte géante à la châtaigne, tartelettes aux lentilles appelées "lentillons", soupe aux choux ou aux champignons) ponctuent cette fête villageoise gourmande. D'autres événements gourmands, comme la "Fête des Bœufs de Pâques ${ }^{22}$ " (le dimanche des Rameaux), dont le boucher René Chatelard est le principal organisateur, mobilisent tout le village (parents d'élèves, commerçants, producteurs etc.).

Les acteurs économiques locaux jouent donc un rôle essentiel pour ériger les produits locaux en marqueurs culturels et identitaires (Bessière, 2006), à l'exemple des 
champignons, mais aussi de la lentille verte du Puy, pour laquelle Régis Marcon s'est battu pour l'obtention de l'AOC dans les années 1990: on la trouve sur les cartes des restaurants du village, dans la boulangerie-pâtisserie (sous la forme de pain aux lentilles ou de confiture de lentilles), ou chez le primeur Joseph Pacalon (La Maison de l'ail).

\section{Conclusion}

À travers cet exemple, nous avons montré en quoi la réputation d'un restaurant triplement étoilé a impulsé une dynamique de développement local qui a bénéficié à l'ensemble du territoire environnant, valorisé par une image attrayante construite sur le localisme alimentaire. L'action de Régis Marcon - que d'aucuns dans le village appellent affectueusement "Régis» ou avec révérence «Monsieur Marcon»- en faveur des produits alimentaires locaux a eu un impact économique, culturel et social sur l'ensemble du village grâce à la mobilisation des autres acteurs locaux.

La concentration atypique de commerces alimentaires de qualité à Saint-Bonnet-le-Froid a été favorisée par des réseaux de solidarité familiale et villageoise et par la proximité de professionnels et d'artisans qualifiés fournissant ou collaborant avec le chef étoilé. À l'échelle locale, ce que Régis Marcon a entrepris pour la valorisation culinaire et culturelle des ressources alimentaires, à l'exemple des champignons (au travers de la cueillette, des recettes, de l'information mycologique), suscite un engouement collectif dont les effets économiques, mais aussi sociaux et culturels sur le village sont positifs. La valorisation des particularismes alimentaires locaux (Poulain, 2002) entraîne ici un cercle vertueux qui tire parti de la «touristification » de nos sociétés, dont le rêve nostalgique (Assouly, 2004) d'une nature et d'une ruralité idéalisées est l'un des fondements.

\section{BIBLIOGRAPHIE}

ABERT J.-F., ANDRÉ P., 2000. La cuisine chez Régis Marcon, Clermont-Ferrand, Éditions du Miroir, $308 \mathrm{p}$.

Assouly O., 2004. Les nourritures nostalgiques : essai sur le mythe du terroir, Arles, Éditions Actes Sud, $143 \mathrm{p}$.

BAilly A., PAELINCK J., 1990. Restaurants, espace, temps, Les cahiers du tourisme, série L, $\mathrm{n}^{\circ} 137$, Centre des hautes études touristiques de l'université de droit, d'économie et des sciences d'AixMarseille, p. 1-32.

BAILly A., Hussy C., 1991. La diagonale gourmande, Mappemonde, n 2, p. 48-49.

BEAUDET G., 2006. La géographie du tourisme gourmand, Téoros, vol. 25, n 1, p. 10-14.

BESSIÈRE J., 2006. Gastronomie de terroir et tourisme, Revue Espaces, n 242, p. 16-21. 
CoSGROVE D., [1984], 1998. Social formation and symbolic landscape, Madison, University of Wisconsin Press, $332 \mathrm{p}$.

CSERGO J., 1996. L'émergence des cuisines régionales, dans FlandRIN J.-L., MONTANARI M. (dir.), Histoire de l'alimentation, Paris, Éditions Fayard, p. 823-841.

ETCHEVERRIA O., 2004. Existe-t-il un goût de l'Aubrac ?, Géographie et cultures, n 50, p. 63-75.

KNAFOU R. (dir.), 2002. Tourismes 1. Lieux communs, Paris, Éditions Belin, collection

« Mappemonde », $320 \mathrm{p}$.

KNAFOU R., 2008. Entre lieux et pratiques : réflexions d'un gastronome amateur, dans CSERGO J., L EMASSON J.-P. (dir.), Voyages en gastronomies, Paris, Éditions Autrement, p. 127-135.

FISCHLER C., 2001. L'homnivore, Paris, Éditions Odile Jacob, 440 p.

HouÉE P., 2001. Le développement local au défi de la mondialisation, Paris, Éditions de L'Harmattan, $250 \mathrm{p}$.

LEMASSON J.-P., 2006. Penser le tourisme gourmand, Téoros, vol 25, n 1, p. 3-4.

LeVY J., Lussault M. (dir.), 2003. Dictionnaire de la géographie et de l'espace des sociétés, Paris, Éditions Belin, $1034 \mathrm{p}$.

MARcon R., MARcon J., JARRy E., LAGer-BARRuel L., 2009. La cuisine de Régis et Jacques Marcon, Paris, Éditions De Borée, collection « Beaux livres ", 244 p.

MATtEUdi E., 1997. Structures familiales et développement local, Paris, Éditions de L'Harmattan, 334 p. MeSPLEDE J.-F., 2004. Trois étoiles au Michelin, Paris, Éditions Gründ, 272 p.

Pecqueur B., 2000. Le développement local, Paris, Éditions Syros, 132 p.

PoulaIN J.-P., 2002. Sociologies de l'alimentation, Paris, PUF, coll. « Sciences sociales et sociétés », $286 \mathrm{p}$.

RoBAGLIA S., [1935], 2002. Margaridou. Journal et recettes d'une cuisinière au pays d'Auvergne, Nonette, Éditions Créer, 230 p.

VIOLIER P., 2008. Tourisme et développement local, Paris, Éditions Belin, 191 p.

\section{NOTES}

1. Au cœur du Haut-Vivarais, Lalouvesc est un important centre de pèlerinage. Sainte Thérèse Couderc en 1826 y fonda la congrégation des sœurs du Cénacle. L'œuvre, née du pèlerinage est aujourd'hui mondiale. Son corps repose dans la chapelle du Cénacle. La chapelle Saint-Jean-François Régis est dédiée à Régis (son nom de famille va devenir grâce à lui un prénom) qui, alors que la France sortait des Guerres de religion, prêcha sans relâche dans les montagnes du Vivarais, des Cévennes et du Velay. Il mourut de froid et d'épuisement en 1640 à Lalouvesc. Saint Jean-François Régis est patron des jésuites de la province de France.

2. A. Bailly et J. Paelinck (1990) distinguent le «restaurant familial» du «restaurant luxueux ». La première catégorie est caractérisée par une localisation aléatoire et une clientèle locale tandis que la seconde est caractérisée par une localisation en fonction de la proximité/accessibilité d'une clientèle potentielle fortunée et de la part importante de 
la clientèle lointaine. Le prix, mais aussi le degré de théâtralité (lié à l'ambiance, à la qualité du service, à la tenue des « hôtes ", à la présentation des plats et à la sélection des produits), sont plus élevés dans le « restaurant luxueux ».

3. Terme utilisé à plusieurs reprises par Régis Marcon lors de notre entretien. Dans son lexique intitulé L'esprit en quelques mots, Régis Marcon écrit à propos de la "nature »: "Sans sa proximité, impossible de survivre... alors je la mets dans votre assiette ».

4. Nous proposons la notion de «lieu symbolique » en référence à celle de "paysage symbolique ", définie comme une "manière de voir " (Cosgrove, [1984], 1998), une idéologie qui révèle comment les groupes sociaux dominants se représentent le monde à travers une relation imaginaire avec leur environnement.

5. En réponse à la question "qu'est-ce qui a déterminé le choix de la localisation du restaurant?", Régis Marcon a écrit "je suis originaire de ce village ». Lors de notre entretien, Régis Marcon a insisté à plusieurs reprises sur son attachement local.

6. Phrase prononcée par Régis Marcon durant notre entretien. Il insiste beaucoup sur «l'esprit d'équipe». Dans son lexique intitulé L'esprit en quelques mots, Régis Marcon définit Saint-Bonnet-le-Froid comme le « berceau familial mais aussi un village animé par une cohésion qui fait avancer les choses ».

7. L'équipe de recherche MIT (Mobilités, Itinéraires, Territoires) a mis en évidence quatre motivations principales du touriste : le repos, la découverte, le shopping et le jeu. Les équipements mis en place par Régis Marcon répondent à trois attentes (le repos, la découverte, le shopping).

8. «Les restaurants sont à la fois des lieux de gastronomie et des lieux qui racontent un pays " (Régis Marcon lors du Café géographique du 23 septembre 2009 à Lyon sur la question « terroir et territoire : vers un patrimoine gastronomique hors-sol ? », animé par le chef étoilé et le géographe Rémy Knafou) [http://www.cafe-geo.net/article.php3? id_article=1662].

9. Notamment les champignons, les herbes et les fleurs. Les plantes sauvages, les herbes et les fleurs, sont à la fois utilisées dans les salades, dans les farces, les sauces, dans les pâtisseries, les infusions (etc.), mais aussi pour la décoration des tables.

10. Appelé aussi « fenouil des Alpes », la cistre a un goût d'anis et de céleri.

11. «Margaridou » était une paysanne auvergnate, qui avait élaboré des recettes au début $\mathrm{du} \mathrm{Xx}^{\mathrm{e}}$ siècle, consignées dans son journal. Celui-ci fut redécouvert et rendu célèbre dans les années 1930 grâce à la romancière S. Robaglia, qui le publia sous le titre Margaridou. Journal et recettes d'une cuisinière au pays d'Auvergne (Robaglia, 2002).

12. [http://www.otmontfaucon.fr/office-de-tourisme].

13. Entretien avec Cécilia Guillien, coordinatrice à l'ALT Pays de la jeune Loire et ses rivières, février 2011.

14. La «Semaine Gourmande» du Haut-Lignon (23 au 31 octobre 2010), les « Vignandises » d'Aurec-sur-Loire (30-31 octobre 2010), la « Foire aux Champignons » de Saint-Bonnet-le-Froid (6-7 novembre 2010), les «Gastrôleries » de Monistrol-sur-Loire (20-21 octobre 2010), le « Marché de Noël et du Terroir » de Saint-Sigolène (4-5 décembre 2010), le « Marché au Foie Gras » d'Yssingeaux (19 décembre 2010).

15. Dossier de presse ALT Pays de la jeune Loire et ses rivières, automne 2010.

16. Entretien avec l'office de Tourisme de Montfaucon, février 2011. 
17. Entretien avec Geneviève Barrière, guide des visites de Saint-Bonnet-le-Froid organisées par l'Office de Tourisme de Montfaucon, février 2011.

18. Ils ont repris les activités (bar-restaurant/boucherie) de leurs parents. Depuis 1986, René a repris l'activité de boucherie, qui emploie aujourd'hui trois salariés en plus de René et Solange Chatelard. Il sélectionne les bêtes chez des éleveurs locaux (Montregard, Saint-Julien, Saint-André, Chaudeyrolles, etc.), avant de les envoyer à l'abattoir à Yssingeaux le lundi et d'avoir la viande en boucherie le mardi.

19. Entretien téléphonique avec Cécilia Guillien, coordinatrice à l'ALT Pays de la jeune Loire et ses rivières, février 2011.

20. Diaporama du lancement officiel de la stratégie «Bon et Bien Manger », ALT Pays de la jeune Loire et ses rivières, 21 février 2011.

21. Entretien téléphonique avec Cécilia Guillien, coordinatrice à l'ALT Pays de la jeune Loire et ses rivières, février 2011.

22. Au cours de la journée, les différents commerces de bouche organisent des dégustations (vins, fromages, pains etc.) et les restaurants proposent des menus thématiques autour de la viande de bœuf.

\section{RÉSUMÉS}

Régis Marcon, chef triplement étoilé au Guide Michelin, est à l'origine d'une dynamique de développement local par le tourisme gourmand dans le village de Saint-Bonnet-le-Froid, à la limite entre la Haute-Loire et l'Ardèche. Son choix délibéré de rester dans son village natal et d'y proposer une restauration de luxe repose sur une valorisation des ressources alimentaires locales. En privilégiant les circuits courts dans son approvisionnement et en bâtissant sa réputation gastronomique sur la promotion du localisme culinaire, Régis Marcon a revitalisé l'économie locale en s'appuyant sur des réseaux de solidarité familiale, économique et sociale.

The Michelin three-stars chef Régis Marcon has impulsed food tourism as a local development strategy in Saint-Bonnet-le-Froid, a village at the border of Haute-Loire and Ardèche (in the eastern part of the French Central Massif). Regis Marcon chose deliberately to set his luxurious restaurant in his native village and to cook in a local fashion. He has built his gourmet reputation through a local food system and through culinary localism, developing the local economy based on familial, economic and social networks.

\section{INDEX}

Keywords : nature, food tourism, Three stars restaurant, family strategy, local development, culinary localism 


\section{AUTEUR}

\section{VINCENT MARCILHAC}

Laboratoire Espaces, Nature et Culture (ENeC) UMR 8185 CNRS (Université Paris 4 - Sorbonne), 190-198 avenue de France - 75013 Paris, France Vincent.Marcilhac@paris-sorbonne.fr 